\title{
¿A más cuerpo, menos sujeto? Ecos de una experiencia en salas de rehabilitación ${ }^{1}$
}

\author{
More body, less subject? \\ Echoes of an experience in rehabilitation rooms
}

\author{
Ps.Cinthia Chufeni
}

\begin{abstract}
Resumen
La pregunta por lo que hace un psicoanalista con el cuerpo de un paciente en las salas de rehabilitación da lugar a la construcción de un problema clínico. Ante el encuentro con un límite físico, se observa una predominancia del cuerpo que va en desmedro del sujeto. En este artículo se exploran el concepto de cuerpo para el psicoanálisis, los paradigmas en discapacidad y los fundamentos de la rehabilitación como disciplina. En este campo, la clínica psicoanalítica resulta oportuna, ya que la dimensión de lo biológico implica al sujeto. Se concluye que incorporar la variable subjetiva brinda un aporte relevante para los tratamientos de rehabilitación.
\end{abstract}

\section{Palabras clave}

Cuerpo - Discapacidad - Psicoanálisis - Rehabilitación

\section{Abstract}

The question about what a psychoanalyst does with the body of a patient in the rehabilitation rooms gives an opportunity: to place a clinical problem. Faced to a physical limit, it's evident that the prevalence of the body is detrimental to the subjectivity. In this article the concept of body is explored through the psychoanalysis, the disability paradigms and the basis of the rehabilitation as a discipline. In this field, the psychoanalytic clinic turns out to be useful, since the dimension of the biological issue implies the subject. It's beeing concluded that to involve the subjectivity, offers a relevant contribution to the improvements of rehabilitation treatments.

\section{Keyowrds}

litation

Body - Disability - Psychoanalysis - Rehabi-

\section{1- Introducción}

Entre los años 2002 y 2007, en las salas de rehabilitación de los hospitales "Juan B. Alberdi" y "Roque S. Peña", pertenecientes a la Secretaría de Salud Pública de la Municipalidad de Rosario, se incorporó un psicoanalista a los equipos interdisciplinarios a cargo de los tratamientos de pacientes con dolencias principalmente motoras. Hasta ese momento, en los equipos predominaban profesionales que sostenían el discurso de las ciencias médicas.

\footnotetext{
${ }^{1}$ El presente artículo se desprende del trabajo final de mi autoría de la Carrera de Especialización en Psicología Clínica, Institucional y Comunitaria: "El enigma del cuerpo. Consideraciones acerca de una experiencia en salas de rehabilitación" (2008), bajo la dirección de la Dra. Cecilia Gorodischer.

${ }^{2}$ Egresada de la Facultad de Psicología, UNR. Especialista en Psicología Clínica, Institucional y Comunitaria. Miembro Equipo Matricial de Discapacidad de la Dirección de Salud Mental de la Municipalidad de Rosario. Argentina.E-mail: cchufeni@hotmail.com
} 
El impacto que produjo ese encuentro nos llevó a formular algunas preguntas, así como también exigió convertirlo en un problema: ¿Qué es lo que se rehabilita? ¿Es el cuerpo? ¿Las intervenciones se dirigen sólo a los cuerpos? ¿Cómo pensamos el cuerpo y la rehabilitación? ¿Cuáles son los efectos en la subjetividad ante la enfermedad, la discapacidad y el dolor? ¿Qué diálogo es posible entre el psicoanálisis y las ciencias médicas? Y la más punzante, ¿qué hace un psicoanalista con el cuerpo de un paciente en rehabilitación?

Secuelas neurológicas, parálisis, pérdidas de funciones y facultades, amputaciones, traumas corporales muestran el encuentro con un límite físico. Estábamos frente a un problema clínico, y su abordaje dependía de cómo se lo leyera.

Una dificultad fue, entonces, la de tomar posición frente a la encrucijada que constituyen el cuerpo y el psiquismo. Efectos de una dimensión en la otra es la regla. Una infección, como caso paradigmático de un proceso biológico, impacta en el narcisismo de un paciente tanto como el curso de una depresión produce marcas físicas en otro.

Nos topamos, también, con la necesidad de repasar conceptos ineludibles, como el de discapacidad y sus paradigmas; $y$, a partir de ahí, explorar la rehabilitación como campo de prácticas.

El presente trabajo es un intento de recuperar partes de aquella experiencia -desde la práctica clínica que pudimos sostener- y reflexionar acerca de ellas, ponerlas en juego, usarlas, como a las piezas de un tangram, para construir algo nuevo.

\section{2- ¿Cuerpo teórico?}

Parafraseando a Freud (1937), comenzaremos diciendo que lo biológico cumple el papel de la roca de origen subyacente para lo psíquico. Pero si el cuerpo es lo que resulta del encuentro de un organismo con el lenguaje, lo corporal no se agota en lo biológico. Para Carpintero (1999), el cuerpo se constituye en un entramado de tres aparatos: un aparato psíquico, sometido a la lógica de los procesos primario y secundario; un aparato orgánico, con sus leyes físico-químicas, anátomo-fisiológicas; y un aparato cultural, regido por leyes económicas, políticas y sociales. Entre los dos primeros hay una relación de contigüidad; pero entre éstos y el último, una relación de inclusión. Ello implica que el organismo no sostiene a lo psíquico ni la cultura es algo externo, la subjetividad se constituye en la intersubjetividad.

¿Qué es el cuerpo para o desde el psicoanálisis? Un enigma que no deja de interrogarnos. Ya en 1925, Freud (1925) advirtió, a partir de la diferencia sexual, de las consecuencias psíquicas de lo anatómico. Desde entonces, la relación entre soma y psiquis se reeditó en múltiples versiones teóricas. En el diccionario de Laplanche y Pontalis (1971), un clásico cuya primera edición data de 1968, el término cuerpo no aparecía como lema. Sin embargo, en otro diccionario de psicoanálisis editado treinta años después (Chemama y Vandermersch, 2004) el cuerpo había obtenido su propia entrada.

La definición de cuerpo y su tradicional oposición a psiquismo fueron revolucionadas por la introducción de los conceptos freudianos; posteriormente, la elaboración que hizo Lacan de los conceptos de cuerpo propio, imagen especular, cuerpo real, cuerpo simbólico, cuerpo de los significantes y objeto $a$, volvieron a producir una profunda transformación (Chemama, 1998).

Pensemos en las diversas teorías del cuerpo; si bien muchas son interesantes y eficaces, ninguna lo dice todo: lo real del cuerpo se les escapa, por la estructura misma del mundo y de las ciencias. Lo real del cuerpo está constituido por todo lo que de él escapa a las tentativas de imaginarización y de simbolización (Chemama, 1998).

El enigma del cuerpo presenta especifici- 
dades cuando lo encontramos en las salas de rehabilitación. Una vez más, la pregunta es ¿qué hacer con él? ¿Cómo acceder al sujeto por la vía del cuerpo? Hay un primer contrapunto que considerar (Wasermann, 1995), el que se produce entre el cuerpo libidinizado el cuerpo erótico, el de los eternos problemas de la sexualidad, aquel con el que se puede bromear- y el cuerpo enfermo -ese de las radiografías, con el cual el humor es más difícil de introducir-.

El vértice de acceso a lo biológico es distinto al vértice de acceso a lo inconsciente. ¿Serían dos vértices de una misma figura? Esa figura hecha de fragmentos unificados por lo psíquico, ¿no es uno de los nombres del cuerpo? Frente a la coexistencia de lógicas orgánicas y psíquicas, nuevamente, ¿por dónde entrar? Por ejemplo, frente al dolor, tanto la respuesta de la analgesia como la de la escucha pueden aliviar. Nos encontramos con dos vías de acceso al cuerpo. Pero intuimos que, si predomina una, se opaca la otra.

Sabemos que son los mismos órganos los que están a disposición de las pulsiones sexuales y de las pulsiones del yo. La boca sirve para besar tanto como para comer y hablar; los ojos no perciben solamente las variaciones de los objetos al servicio de la conservación de la vida, sino también los rasgos que condicionan la elección amorosa. Cuanto más íntima sea la relación que un órgano contraiga con una de las funciones, más se rehúsa a la otra (Freud, 1910).

Este postulado acerca del incremento de una función en detrimento de la otra nos resultó crucial para aceptar la siguiente sospecha: ¿a más cuerpo, menos sujeto? Tomaba forma una hipótesis: la de la relación inversamente proporcional entre organismo y psiquismo. Éste fue un punto de insistencia en el recorrido de nuestra experiencia en las salas de rehabilitación, donde nos topamos con el cuerpo como sangre, como desgarro; un tema que se prefiere evitar, atribuyéndolo al campo de la medicina (Waserman, 1995). Sin embargo, ese cuerpo desarmado habita como amenaza en las fantasías de cada hablante.

En las salas de rehabilitación, el cuerpo se presenta herido, dañado, con discapacidades. ¿Qué efectos subjetivos encontramos cuando la amenaza se convierte en un hecho consumado? Contamos, para empezar, con la premisa clínica de que la enfermedad no impide el fantasma. Por el contrario, un proceso orgánico es ocasión de que las fantasías se encastren, singularizando ese padecimiento, pero, a la vez, parasitando la palabra posible (Raimbault, 1985).

Se volvía necesario, entonces, abordar el cuerpo en clave metapsicológica; esto no significaba darle ese estatuto, sino pensarlo como un fondo tocado por la exploración de los procesos psíquicos en momentos decisivos, sobre todo en los registros pulsional y narcisista (Assoun, 1994).

Revisemos el campo semántico del cuerpo freudiano: Freud emplea varios términos. En alemán, cuerpo es Körper, cuerpo real, objeto material y visible, extenso en el espacio y designable por cierta cohesión anatómica; pero también cuerpo es Leib, cuerpo aprehendido en su propia sustancia viva, no solamente un cuerpo sino el cuerpo, principio de vida y de individuación. Por último, el cuerpo remite al registro de lo somático (somatisches), adjetivo que describe procesos determinados que se organizan de acuerdo con una racionalidad. De este doble estallido -conceptual y temático-, se desprende una nueva imagen de la corporeidad revisada por lo inconsciente.

El cuerpo se anuncia con una paradoja: designa a la vez una profundidad y una superficie. En el plano terminológico, esta distinción podría coincidir en parte con la de Leib y en parte con la de Körper.

Habría una instancia del cuerpo pre-metapsicológica: la pulsión, concepto límite entre lo psíquico y lo somático. La pulsión encuentra su fuente en un lugar somático por 
la excitación, que se traduce en una tensión originaria; pero la moción correspondiente se manifiesta como psíquica, y tiende a la supresión del malestar por mediación de un objeto.

Existe, en efecto, el cuerpo como soporte de funciones corporales necesarias para la vida; pero, mediante la satisfacción de las necesidades, lo que se instala es un cuerpo erótico, el cuerpo del síntoma. ¿Cómo articular esta doble entidad del cuerpo? Assoun (1994) propone una relación metafórica entre los dos cuerpos. A partir de un fenómeno físico de modificación corporal - la entrada en la enfermedad-, se despierta el cuerpo del síntoma psíquico; suele ocurrir que una alteración funcional mórbida pase a representar fantasías inconscientes que acechaban la primera ocasión de manifestarse. Esto podría constituirse en razón suficiente para pensar la pertinencia de la práctica psicoanalítica en las salas de rehabilitación.

Retomando el desarrollo metapsicológico, no hay en la obra freudiana una teoría del esquema corporal porque el cuerpo no es una función, sino que es promovido como soporte de la función narcisista. El sujeto toma a su propio cuerpo como objeto de amor. El cuerpo propio está en el lugar del sí mismo.

Assoun (1994) afirma que lo que más nos acerca a la teoría freudiana de lo corporal es el Yo de la segunda tópica: un yo que sería cuerpo, lugar del que pueden provenir, simultáneamente, percepciones externas e internas. El yo es, para Freud (1923), la proyección de una superficie. El cuerpo interviene en la génesis del yo y el yo está estructurado como el cuerpo, a la vez límite y extensión (Assoun, 1994).

El yo sería la subjetivación de la superficie corporal; aunque "es menos el producto de una experiencia corporal que el acontecimiento de la aparición del cuerpo como propio" (Assoun, 1994: 254).

En principio, el cuerpo se nos presentó como un campo ajeno a la práctica del psi- coanálisis; pero esa impresión quedó revisada a partir del cuerpo teórico del mismo. La pregunta clínica que se impone es qué podríamos hacer frente al cuerpo de un paciente, ese cuerpo del que se habla. Llegamos a las salas de rehabilitación disponiendo de la escucha y la palabra como instrumentos, ¿para hacer intervenciones en el cuerpo?

\section{3- Puntos panorámicos: los paradigmas en discapacidad}

En el trabajo de exploración teórica realizado a partir de la incorporación a las salas de rehabilitación, encontramos una serie de paradigmas operantes respecto de la discapacidad. Recordemos que la mayoría de los pacientes de dichas salas tienen discapacidades motoras genéticas, congénitas o adquiridas a lo largo de la vida por accidentes o enfermedades.

En tal sentido, se hizo necesario relevar el lugar que le daba a la diferencia cada paradigma -el tradicional, el médico o rehabilitador y el social-, ya que, por antagónicos que parezcan, coexisten en las prácticas, en cada tratamiento, en las instituciones y sus lógicas y en las posiciones de los profesionales como actores sociales. Foucault (1996) plantea que cada sociedad genera mecanismos de percepción de lo diferente y modos de tratarlo. Hay una construcción social de la discapacidad (Pantano, 1987) que se evidencia, por ejemplo, en el vocabulario. Lo que se percibe como débil, deficiente, inválido genera posiciones que tienden a fortalecer, compensar, validar.

Lo que encontramos dentro de lo que se llamó el primer paradigma es una visión social tradicional y discriminatoria. Historias como la de Esparta, en la que los niños con malformaciones eran sacrificados, y que representan una visión extrema de las prácticas de descarte de lo diferente, están inscriptas en nosotros y podríamos decir que tienen vigencia en los prejuicios. Las personas con discapacidad no responden a los estereotipos de normalidad 
y, como resultado de significaciones y pautas culturales muy arraigadas, son objeto de acciones estigmatizantes de forma cotidiana.

En los siglos XV y XVI, con el surgimiento de los estados modernos y el ordenamiento racional y administrativo de los súbditos, los diferentes pasaron de ser asistidos a ser controlados. Y, en el siglo XIX, con el positivismo, se convirtieron en objetos de estudio científico.

El segundo modelo que consideramos fue el paradigma de la rehabilitación. Las salas han sido el gran dispositivo de esta concepción surgida en el siglo XX como consecuencia de las condiciones en las que quedó el proceso de desarrollo industrial tras las dos guerras mundiales. La posguerra dejó sus secuelas: un gran número de lisiados por los que el Estado tuvo que responder. Fue así como se incorporó a la medicina una nueva práctica: la rehabilitación, y con ella, la discapacidad pasó a ser percibida como un asunto sanitario y como un daño a reparar. Desde una posición clásica, la rehabilitación estuvo regida por una serie de objetivos: el uso básico de las manos, la ambulación, la independencia en la atención personal, la comunicación, el lograr una apariencia en lo posible normal y la restitución al bienestar (Cibeira, 2006).

La especialidad médica dedicada exclusivamente a esta materia desde 1936 es la Medicina Física o Fisiatría; su tarea es valorar, restaurar y conducir el tratamiento de pacientes con discapacidades crónicas neuromusculoesqueléticas y circulatorias. La rehabilitación nació, entonces, con la aparición de las secuelas invalidantes que causaban deficiencias. Lejos de aquellas guerras, la batalla contra la poliomielitis enseñó a los argentinos otra razón de ser de la rehabilitación.

En 1969, la OMS (Organización Mundial de la Salud) redefinió a la rehabilitación médica como la asistencia que trata de desarrollar las capacidades funcionales y psicológicas del individuo y sus mecanismos de compensación para su desarrollo autónomo y activo. El obje- tivo de la rehabilitación médica -a diferencia de lo que proponía la definición tradicionalpasó a concentrarse en el tratamiento y medición de parámetros funcionales relativos a la movilidad, el cuidado de sí mismo, la habilidad manual y la comunicación.

Más tarde, aparecieron leyes de integración social de los minusválidos y la rehabilitación comenzó a ser percibida como un derecho. En este marco, el problema era definido como individual; la deficiencia era el origen de las dificultades, y la solución, tanto como el control del proceso, estaba en manos de la intervención profesional del equipo rehabilitador. El éxito se medía en función de las destrezas alcanzadas por los pacientes; entre las cuales estaba la obtención de un empleo o un cuerpo productivo. El modelo industrialista de la rehabilitación fue superado, por ejemplo, por las escalas de Fugl-Meyer, que agregó a lo motriz índices de evaluación más amplios: el autocuidado, la capacidad de ocio, la capacidad profesional, la vida social, matrimonial y sexual, la situación financiera.

Fue en este contexto que la OMS propuso la Clasificación Internacional de Deficiencias, Discapacidades y Minusvalías, que si bien son conceptos que hoy atrasan, han significado un aporte a la reflexión sobre estas problemáticas. Las definiciones presentaban una serie de distinciones que aún resultan útiles. Por un lado, la deficiencia se refería a las anormalidades de la estructura corporal y de la apariencia y de la función de un órgano o sistema. Las discapacidades, en cambio, reflejaban las consecuencias de la deficiencia desde el punto de vista del rendimiento funcional y de la actividad del individuo; se hablaba de trastornos de la persona. Finalmente, las minusvalías eran entendidas como las desventajas experimentadas por el individuo como consecuencia de las deficiencias y discapacidades, y reflejaban el impacto en la interacción y adaptación al entorno (Bellacasa, 1990).

Tras el auge de estos paradigmas, el tradi- 
cional y el médico o rehabilitador, se impuso un nuevo paradigma o modelo social sobre la discapacidad, surgido en las últimas décadas y construido desde la perspectiva de los derechos humanos. Desde este enfoque, las distintas discapacidades son consideradas una característica más dentro de la diversidad de la población. Este paradigma tiene un antecedente político en el movimiento social de la autonomía personal, que trasciende la frontera de los tratamientos; se trata de un horizonte impulsado por los mismos individuos afectados, con un carácter contestatario a las estructuras ya establecidas. Son las propias personas con discapacidad quienes buscan construir su autonomía, lejos de las prescripciones médicas. Su eslogan da sobrada cuenta de esa posición: Nada sobre nosotros sin nosotros. Durante el transcurso de nuestra experiencia en las salas de rehabilitación, se trataba de un modelo vigente en la teoría, pero incipiente en las prácticas.

Las políticas públicas, marcos legales y normativas actuales en la República Argentina se han encaminado a considerar a la discapacidad como una cuestión de derechos humanos, basadas en el paradigma social. En el año 2008, nuestro país sancionó la Ley 26.378, que ratifica a la Convención sobre los derechos de las personas con discapacidad.

Allí se definió a la discapacidad como el resultado de la interacción entre las personas con deficiencias y las barreras que existen en la sociedad. De esta nueva definición, se desprende que la discapacidad sólo existe si una persona con una deficiencia no puede realizar una actividad específica. Es decir, si no hay una barrera física, actitudinal o ideológica, no hay experiencia de la discapacidad. Un ejemplo sería el de una persona con silla de ruedas usando un ascensor; en esa escena, al sortearse el obstáculo de la escalera, la discapacidad no se produce.

A partir de este cambio conceptual la discapacidad queda desontologizada. ¿Qué se rehabilita, entonces? ¿Lo rehabilitable sería el cuerpo en tanto portador de la deficiencia orgánica? Responder nos obliga a hacer aclaraciones. Por un lado, como ya señalamos, el cuerpo excede a lo orgánico. Además, no pensamos en una rehabilitación estrictamente médica, sino en una más amplia.

Actualmente, no se considera que la rehabilitación agote el universo de las acciones en discapacidad, ya que se trata de una problemática que alcanza el campo de los derechos y de la escena social. Entendemos que el desafío pasa por lograr que los tratamientos, la atención de las personas con discapacidad, tengan en su horizonte estas nuevas perspectivas.

\section{4- La sala en escena}

Históricamente, la admisión de los pacientes a los servicios de rehabilitación consistió en una consulta con el médico fisiatra, durante la cual se realizaba una evaluación y se determinaba un plan que consistía en indicaciones -medicamentos, estudios, prótesis, ortesis- y en la derivación a especialidades o disciplinas asociadas a la rehabilitación. Este modelo ha ido cambiando y ha dado lugar a admisiones interdisciplinarias que implican, por ejemplo, la presencia simultánea de un médico, un psicólogo y un trabajador social. Además, la discusión de los planes de rehabilitación comenzó a tener lugar en las reuniones de equipo. La rehabilitación ha sido una disciplina muy resistente a la democratización. Años de historia de hegemonía del discurso médico hacen de contrapeso a la novedad de la rehabilitación basada en la comunidad.

En las salas de rehabilitación, fuimos testigos de muchas escenas impregnadas de prejuicios y modelos conservadores que ubicaban a las personas con deficiencias como enfermas, recluidas, infantilizadas, asistidas o protegidas de por vida, sin autonomía. Objeto de miradas que generaban prácticas teñidas por el rechazo, la tutela y el control.

En los certificados de discapacidad actua- 
les, la palabra rehabilitación es una prestación que hace referencia a los tratamientos y apoyos requeridos por la discapacidad acreditada -kinesiología, psicología, terapia ocupacional, acompañamientos terapéuticos, entre otros-. Durante la experiencia cuyos ecos recuperamos en este texto, en cambio, hablábamos de rehabilitación para referirnos, específicamente, a la rehabilitación motriz. Así como la discapacidad excede a los cuerpos, la rehabilitación salió de las salas, y hoy se agregan, en los proyectos terapéuticos, estrategias externas a los efectores de salud -como la asistencia personal en el hogar y en el espacio social, entre otros apoyos-.

En 1982, el Programa de Acción Mundial para las Personas con Discapacidad de la ONU (Organización de las Naciones Unidas) definió a la rehabilitación como un proceso de duración limitada y con un objetivo determinado, encaminado a permitir que una persona con deficiencia alcance un nivel físico, mental y social funcional óptimo, proporcionándole así los medios para modificar su propia vida. Esta noción comprende medidas orientadas a compensar la pérdida de una función o una limitación funcional y otras destinadas a facilitar ajustes o reajustes sociales.

Durante nuestra experiencia, los equipos que integramos adherían a esta definición oficial. En cuanto a la duración limitada, en los casos de tratamientos de procesos agudos, se trataba de una meta que no presentaba dificultad alguna. La mayoría de los pacientes, no obstante, padecía de afecciones crónicas, por lo que resultó fundamental no confundir el carácter crónico de la afección con la cronificación de los tratamientos.

En el objetivo de alcanzar un nivel físico, mental y social óptimo, resuena, por otra parte, aquella idea de restitución del bienestar presente en las definiciones históricas de la rehabilitación. Freud argumentó la imposibilidad de un bienestar en la cultura y, desde nuestra posición como psicoanalistas, no podíamos no cuestionar esta ilusión. Por ello, intentamos transmitir que un resultado óptimo era, ante todo, algo que además de incluir un límite era a construir cada vez entre los pacientes y los profesionales. En ocasiones, al interrogar a los pacientes acerca de qué esperaban de la rehabilitación, escuchábamos que se pretendía obtener lo que se había perdido; incluso más de lo que había antes del accidente, ya que el cuerpo perdido también era -aunque en menor medida- uno fallado.

Respecto de proporcionar los medios para modificar la propia vida, nuestras intervenciones fueron en el sentido de promover una posición ética en los integrantes de los equipos. Sostuvimos que cada sujeto era dueño de su cuerpo y que, por lo tanto, promover la autonomía era diferente a direccionarla. En consecuencia, frente a cada paciente renovábamos la inquietud de hasta dónde llegar y cómo propiciar la autonomía, aun si no era lo demandado.

Por último, en cuanto a los planos individual y social aludidos en la definición que venimos analizando, fue frecuente el trabajo con el entorno de los pacientes, familiares o figuras de sostén. También se realizaron intervenciones con instituciones para favorecer la inclusión de personas con discapacidad, desde rampas en una escuela hasta promover medidas tendientes a la sensibilización de la población de un club en torno a la problemática de la discapacidad. El acento estaba puesto, sin embargo, en quien padecía los límites; lo que se procuraba era que hiciera algo con lo que le ocurría, y no confundir que la ciencia no pudiera hacer nada con que nada pudiera hacerse.

La tarea de tejer redes no se dirigió sólo hacia el afuera, sino que se realizó también en el interior de la sala. Aunque la interdisciplina tenga estructura de promesa, sostenerla fue un intento cotidiano, nunca garantizado, pero tampoco perdido de vista. En los contornos de las distintas disciplinas y en su encuentro con otras, veíamos producirse un nuevo sa- 
ber. Como resultado de ese espacio de trabajo, arribamos a principios rectores construidos interdisciplinariamente. Si bien no desconocemos la distancia entre éstos y las prácticas, sabemos que sin lineamientos de trabajo, o ante la ausencia de posiciones políticas al respecto, el panorama hubiera sido más desalentador. Para empezar, como integrantes de los equipos de rehabilitación, consideramos esencial enmarcar las acciones en proyectos terapéuticos sin desentendernos de las condiciones sociales, la pertenencia y los recursos materiales y simbólicos de cada sujeto.

Dado que las salas en las que trabajamos se encontraban en hospitales municipales, el marco eran las políticas públicas en discapacidad, cuyos ejes son la búsqueda de la equidad junto con la profundización de la accesibilidad, entendida como la remoción de barreras físicas y de orden cultural e ideológico; ya que las prácticas en salud -como toda práctica social- no están exentas de reproducir barreras disfrazadas de inclusión. Al revisar la atención en salud, se observa que las respuestas de los profesionales constituyen un determinante de las modalidades que los procesos adoptan y condicionan con fuerza la producción de discapacidad. La rehabilitación en las salas se enmarcaba en este proceso y asumía de algún modo la responsabilidad de atenuarlo o revertirlo.

¿Cuáles fueron las condiciones tenidas en cuenta para que la rehabilitación no profundizara el proceso de producción de discapacidad? Oportunidad, pertinencia y sustentabilidad de las intervenciones. Estos tres componentes no se pueden pensar aisladamente. Cada uno da lugar al otro, lo potencia o lo debilita. Para diseñar un plan de rehabilitación, fue orientador preguntarse por lo oportuno para una persona en su propio aquí y ahora. ¿Cuáles eran las acciones pertinentes a realizar? ¿Quiénes poseían los recursos al interior del sistema de salud para llevarlas adelante? ¿Cuáles iban a llevarse a cabo por fuera del sistema de salud?
¿Cómo íbamos a efectivizar las acciones y a sostenerlas en el tiempo? ¿Con qué recursos contábamos y cuáles había que producir?

Por otro lado, un proyecto terapéutico sólo podía adquirir una direccionalidad adecuada al asentarse en la historia de los sujetos; de lo contrario, corría el riesgo de convertirse en la aplicación mecánica de un protocolo; o bien podía tomar la forma de la planificación de la vida de alguien y confundirse con un proyecto de vida. Constatamos que convenía trabajar sobre los obstáculos que se iban presentando en la cotidianidad del paciente ante su nueva situación, sin olvidar que la vida está en otra parte, no en las salas de rehabilitación.

Muchas veces nos topábamos con la resistencia de los pacientes al alta, a dar por terminada la rehabilitación en sentido estricto, porque esto los dejaba con la sensación de quedar sin perspectivas de vida. Quizás los mismos equipos, al reducir la rehabilitación a sus posibilidades técnicas, producían en los pacientes la impresión de un hasta acá llegamos, no se puede hacer nada más. Era necesario, de nuestra parte, intervenir en el sentido de que el límite de la rehabilitación no era el del sujeto.

Respecto del alta, se procuró que, desde el inicio, los pacientes pudieran construir un afuera del dispositivo. Con frecuencia se sentían muy cómodos en las salas y aceptaban sin objeciones las dinámicas de trabajo propuestas; por supuesto, se trataba de lugares adaptados, poblados de pares, con gente entrenada para asistirlos. Pero la clave estaba en generar recursos para el afuera. El después de la rehabilitación tenía que formar parte del proyecto terapéutico. Cuando esta problemática no era abordada, podía tener efectos indeseables, como la negativa de los pacientes a dar por terminados los tratamientos; algunos se sentían abandonados y otros reclamaban que la rehabilitación no habia cumplido sus objetivos. Este contraste entre los objetivos de la disciplina y los de los pacientes daba cuenta de la necesidad de escuchar la voz del sujeto. 
5- Tensión entre psicoanálisis y rehabilitación

En términos generales, respecto de lo que sucedía en la cotidianidad de las salas, las intervenciones eran de lo más variadas, pero predominaban aquellas que consistían en manipulaciones físicas, reeducaciones, indicaciones de prótesis, y otras tantas maniobras sobre el cuerpo. Esto nos obligó a pensar qué más de la rehabilitación nos concernía como psicoanalistas.

Recordemos que, en un primer momento, sentimos a la rehabilitación como un campo ajeno, hasta que comenzamos a visualizar inquietudes que, con trabajo, supervisiones y lecturas, se constituyeron en preguntas orientadoras. Las intervenciones específicas, desde la clínica psicoanalítica, se fueron dando a medida que nos íbamos interiorizando del funcionamiento de las salas; un interior al que intentábamos situar como exterioridad. En todo momento tuvimos presente que el lugar del psicoanálisis respecto de la medicina es marginal, extraterritorial (Lacan, 1996).

La rehabilitación se ofrece como práctica reparatoria, la semántica de la palabra lo insinúa; sostiene ideales desde los que se desconoce que el cuerpo implica malestar. Pensar el cuerpo, las claves de su constitución y su lógica desde el psicoanálisis, supone, en cambio, resistirse a leer un accidente sin una historia y a dirigir un proyecto terapéutico por fuera de lo habitable para un sujeto singular. La rehabilitación completa, universaliza, ausculta el cuerpo; mientras que el psicoanálisis descompleta, singulariza, escucha al cuerpo aún en su silencio.

La lógica de las salas se enmarca en el discurso de la medicina, y la consecuente forclusión del sujeto, que intenta hacer del cuerpo algo completamente descifrable. En la actualidad, los cuerpos parecen haber perdido su condición de enigmáticos. La ciencia avanza y promueve la ilusión de trascender la enfermedad, la vejez y la muerte (Sibilia, 2009). Ór- ganos que se reemplazan, procesos biológicos que se corrigen; límites puestos permanentemente en tela de juicio bajo la forma del desafío o el desconocimiento. Por supuesto, los avances tecnológicos son bienvenidos, ya que han permitido prolongar y mejorar las condiciones de la vida. Sin embargo, es la potencia de lo psíquico lo que iría desdibujándose frente a la nitidez que adquiere el saber acerca de lo somático. Como si la preponderancia de uno fuera en detrimento de lo otro. Nuevamente, a más cuerpo menos sujeto.

Los tiempos que corren, ¿favorecen una versión desubjetivada de la experiencia del cuerpo? ¿Hay un cuerpo de la época, una experiencia del cuerpo signada por la cultura? Hoy la sociedad demanda soluciones rápidas, respuestas generalizadas, sin el tiempo inherente a la formulación de las preguntas singulares. Las neurociencias prometen tips que todos quieren comprar. No recordamos qué venden, no importa: es una ilusión. Frente a la ciencia, el psicoanálisis resiste como uno de los refugios del sujeto, alternativo a la lógica imperante del mercado.

Nos preguntábamos por el modo de reintroducir la subjetividad. Su rechazo es un punto de anclaje de la medicina y condición de su eficacia, ya que esa exclusión es estructural a toda práctica científica. Sin embargo, esto debía diferenciarse de lo que son los modos de cercenamiento de la subjetividad en las prácticas médicas actuales, capitalismo mediante. La práctica médica amenaza llegar a convertirse en una industria y el médico en un técnico o en un engranaje.

A través de las encrucijadas que nos planteó la clínica psicoanalítica en rehabilitación, el cuerpo dejó de ser un campo ajeno para convertirse en fuente de interrogaciones en lo más propio de nuestra práctica. Ante el encuentro traumático con un límite corporal, irrumpe la angustia, nexo entre el cuerpo y las palabras, recurso que posibilita la elaboración y la apertura a las resignificaciones. Era una 
ocasión oportuna para la oferta de un trabajo discursivo; pero también nos enfrentábamos con el riesgo de generar expectativas erróneas, las de lograr un happy end. Por lo tanto, volvimos a preguntarnos por la especificidad de nuestra práctica, de nuestra oferta, de nuestro lugar entre las disciplinas de la salud con las que convivíamos en las salas.

En primera instancia, se trataba de escuchar a cada paciente en su singularidad, después de un accidente, antes de alguna cirugía o bajo el impacto de la noticia de una enfermedad incipiente. En la diversidad de manifestaciones encontramos una insistencia: ante un proceso mórbido bay un debilitamiento subjetivo. Lo que produce la presentificación del cuerpo es la desaparición de la función del sujeto, y ello genera, al mismo tiempo, una posición de inermidad, de desamparo.

Una constante en las derivaciones que recibíamos de médicos y kinesiólogos era la mención a la percepción de una carga extra en los pacientes cuando se referían a sus dolencias; que mostraban una angustia desmedida o que hablaban demasiado, que se quejaban de más o que lloraban mucho; esto siempre según el criterio de los profesionales que indicaban asistencia psi. Frente a un déficit, las derivaciones se pedían por registrar excesos: de angustia, de palabras, de demandas.

En otras ocasiones, se daba una suerte de enloquecimiento ante la enfermedad, sin que esto significara estar frente a la locura. Cuando la libido es modificada por un proceso mórbido, el yo encuentra un mundo loco al que hay que dominar y se comporta, pues, como un yo loco (Rabant, 1993). Tal era la situación de pacientes a los que se tildaba de imposibles, con los que no se podía. A partir de trabajar con ellos, fuimos poniendo en agenda de los equipos el padecimiento subjetivo como algo a incluir en las rehabilitaciones.

Ante una dolencia, hay un cambio en la economía libidinal, y esto tiene consecuencias en el yo; se resigna el interés por aquellos ob- jetos del mundo exterior que no se relacionen con el sufrimiento (Freud, 1915). En un gran número de casos, tomábamos como criterio de recuperación subjetiva que los pacientes restablecieran su capacidad de libidinizar el mundo.

En la obra freudiana es tan grande el abanico de opciones ante la enfermedad, que va desde el estallido de neurosis a propósito de una alteración física hasta la compensación psíquica a partir de una enfermedad, en función de que el aparato se asegura una cuota de sufrimiento, explicado desde la economía del masoquismo (Freud, 1924). En la clínica psicoanalítica en rehabilitación esto fue confirmado en numerosos casos.

Por ejemplo, recibir diagnósticos no siempre traía aparejado un desprendimiento de angustia. Por el contrario, en muchos sujetos tuvo un efecto apaciguador poner nombre $a$ sus males. El discurso médico tuvo, en estos casos, un lugar de auxiliar necesario para la construcción de un cuerpo y una historia. En cuanto a nuestra disciplina, esto cerraba la posibilidad de un trabajo clínico allí donde, en otros pacientes, se posibilitaba una apertura, al posicionarse frente a la enfermedad como un límite que reordena la vida.

Otra escena frecuente en las salas era la invitación peligrosa a empezar de cero. Sostuvimos firmemente que la rehabilitación, en todo caso, podía ser un punto de partida de un nuevo trayecto. Reconocer la existencia de una historia fue condición de que el proceso se constituyera como propio, de que hubiera una rehabilitación con nombre propio, subjetivada.

Un paciente con secuelas de un accidente cerebro vascular (ACV) refería, justamente, que todos le pedían que empezara de cero, que se olvidase de cómo eran las cosas antes, le decían que estaba vivo, que dejara de hablar de la muerte. Sin embargo, él se sentía muerto en vida desde el día del accidente. La vida parecía haberse detenido allí, así que fue invi- 
tado a hablar de aquella frase y, a partir de ahí, empezar a trabajar. En la segunda entrevista, surgió el recuerdo de la muerte de su padre, confirmando que las pérdidas reactualizan no sólo las pasadas, sino, también, la relación que se tiene con las mismas y con el trabajo de duelo. Sólo partiendo de algo propio es posible empezar a rehabilitarse.

Así sucedía, igualmente, con la finalización de los tratamientos. Recibimos una consulta de una mujer con secuelas de polio que, veinte años después, pedía terminar su rehabilitación, a su juicio interrumpida a sus diez años. Sentía que su pierna no había quedado bien, y que esto impedía el avance de su vida según sus deseos. Interrogamos esta certeza acerca de la causa de sus impedimentos. Los médicos evaluaban que su déficit era menor y que sus dificultades se compensaban al caminar, que no había nada que rehabilitar. Sin embargo, dimos lugar a un tiempo de trabajo en el que se pudieron recuperar diversas frases oídas en la infancia diciendo que las cosas no marchaban bien con ella.

En las salas, donde abundaban las parálisis, intentábamos producir movimientos. Y en aquellas situaciones en las que los pacientes pretendían continuar y profundizar las preguntas formuladas, derivábamos esas demandas a otros espacios de salud mental, donde los cuerpos estuvieran más silenciados y ya no avanzaran tanto sobre los sujetos.

Así fuimos respondiendo a la pregunta por lo que hace un psicoanalista en las salas de rehabilitación: dar soporte al trabajo en tensión y mantener la extranjería del psicoanálisis respecto del discurso médico. Por momentos, parecíamos los únicos que trataban con la subjetividad de los pacientes y, a veces, costaba instalar la idea de que éramos todos los integrantes de los equipos quienes íbamos a producir efectos en ese campo. Sin embargo, estar advertidos de lo ilusorio de la pureza de la teoría de la comunicación, saber de la distancia que existe entre lo que se dice y lo que se escucha, saber que el mensaje no es algo acabado previamente a ser enunciado, sino que se produce al ser recibido, poder dirigir las interferencias al atravesamiento inconsciente que nos caracteriza como sujetos fueron recursos que nos permitieron sostener las intervenciones específicas.

El tránsito por esta experiencia nos llevó a aceptar que la rehabilitación puede ser una historia a escribir singularmente. Esta es la propuesta del neurólogo Oliver Sacks (2001) en sus libros; en particular, en Un antropólogo en Marte. Siete relatos paradójicos. Allí se relatan historias de pacientes que compensaron sus déficits mediante lo que Sacks llama la paradoja de la enfermedad, esas respuestas que la medicina no espera y que llevan la marca del sujeto.

\section{6- Momento de concluir}

Al incorporarnos a las salas de rehabilitación, tuvimos la sensación de ingresar en un campo ajeno a nuestra disciplina: el de las intervenciones sobre el cuerpo. Como consecuencia del trabajo realizado, fue posible vislumbrar que tanto los cuerpos como las rehabilitaciones mismas eran impensables sin la dimensión subjetiva. Tener un cuerpo implica una exigencia de trabajo para el psiquismo.

Nuestra pregunta acerca de si era el cuerpo lo que se rehabilitaba quedó reformulada. Si bien la rehabilitación parece acontecer en la dimensión orgánica, nunca es el cuerpo sin el sujeto. Es más, se trata de dos términos que mantienen una relación inversamente proporcional entre sí. Advertidos de su imbricación, el debilitamiento subjetivo como consecuencia de una afección orgánica fue el objeto que vertebró nuestras intervenciones. En cada derivación, en cada interconsulta, fuimos constatando líneas de trabajo que hicieron que el campo de intervenciones nos resultara menos ajeno hasta convertirse en uno propio. La inquietud sobre qué hace un psicoanalista con el cuerpo de un paciente fue cediendo. La forma 
en que se presentaba el cuerpo era inquietante, pero incorporarse en los equipos no fue imposible.

La práctica clínica en salas de rehabilitación mostraba crudamente la fragilidad de lo humano, la encarnación de límites difíciles de soportar, de los que nadie quería saber. Más aún, la vida parecía posible a condición de silenciarlos; lo sabíamos, pero aun así... (Mannoni, 1973). Frente a un límite, renegar de él es una de las posibles respuestas. En distintos momentos de este trabajo, presentamos las prácticas en rehabilitación como reparatorias. Ahora enunciamos su deslizamiento a lo renegatorio, entendido como constatación y rechazo de un límite. La sociedad, en su clave exitista, es subsidiaria de la renegación de los límites, y lleva a vivir los déficits como vergonzantes. Queda pendiente la formulación de nuevas preguntas que incluyan los rasgos de época que exacerban esta versión de la rehabilitación.

En el recorrido de los cambios conceptuales en la materia, situábamos a la autonomía como el eje de las políticas y teorizaciones actuales en discapacidad. Sin lugar a dudas, si bien se trata de algo superador, encierra riesgos. Lo autónomo no es lo autosuficiente. La posición contestataria del poder médico hegemónico y de la sociedad de control, a la que adherimos como horizonte en las prácticas de atención, puede traer aparejada una posible exaltación narcisista: yo soy la única ley de mi vida; en este punto, también, parece que alguna parte del límite no pudiera inscribirse.

Los conflictos en torno a los límites insisten en todos los rincones de nuestro desarrollo. Al happy end, que los desmiente, lo dejamos en otro idioma, porque nos resulta extranjero en su lógica e imposible de traducir en una oferta genuina para nuestros pacientes.

Las prácticas en rehabilitación no deberían permanecer ingenuas frente al orden de cosas que venimos situando. Producto del encuentro con la castración, que es el límite del sujeto, la angustia era inevitable, y las posiciones defensivas que se generaban eran variables subjetivas que afectaban no sólo a los pacientes, sino también a los profesionales, y condicionaban la percepción y direccionalidad de los tratamientos.

Nombramos al trabajo en rehabilitación como una clínica de los límites; aunque probablemente ninguna no lo sea. Subjetivamente, del encuentro con un límite puede resultar un enredo que sería oportuno trabajar con un psicoanalista. El cuerpo es una ocasión privilegiada de ese encuentro, de ese anudamiento, y de un trabajo clínico.

Por otra parte, conviene no favorecer una nueva ilusión. Sumando un consultorio psi, algunos miembros de los equipos renovaban la falsa promesa de completud del tratamiento o la de la rehabilitación integral; pero nuestra posición fue, claramente, en otro sentido. Desde el psicoanálisis, intentamos sostener el reconocimiento de la subjetividad, o responder a la demanda de este reconocimiento, que es del orden del deseo. No podíamos sumar un protocolo, como el resto de las disciplinas intervinientes, ya que así se excluye lo singular, lo específico de nuestra práctica.

Intentamos instalar una ética, respetar los tiempos y las posiciones de los pacientes. Si sólo se tratara de respetar a los pacientes, charía falta un psicoanalista allí? Probablemente, en un intento de humanizar la medicina, a veces, éramos convocados por integrantes de los equipos como asesores del trato a los pacientes. Si bien el reconocimiento del otro en tanto semejante es una primera instancia, con ello aún estamos lejos de producir un reconocimiento de la alteridad, la subjetividad signada por lo otro en cada uno, pacientes y profesionales. Lo rechazado en el otro es lo rechazado y desconocido en nosotros mismos, la castración, el límite.

Por último, nos resultó ordenador ubicar distintos planos de intervención: con los pacientes, por un lado, y con los miembros de 
los equipos, por otro. Con los primeros, pudimos revisar algunos efectos que tienen lugar en la subjetividad a la hora de la enfermedad, ese momento en que el cuerpo se hace particularmente presente para un sujeto y avanza sobre él. Ofrecíamos un espacio de trabajo donde tramitar acontecimientos a partir de la elaboración psíquica frente a la irrupción de lo traumático. En cuanto a los equipos, intervinimos en la línea de insistir en que la subjetividad sea reconocida a la hora de diseñar las estrategias de rehabilitación. También defendimos una ética en el sentido de dirigir los tratamientos y no las vidas de los pacientes. La escritura de esta experiencia suma un tercer plano: el intento de transmitirla.

\section{Referencias:}

- ASSOUN, PAUL LAURENT (1994). Introducción a la metapsicología freudiana. Buenos Aires. Paidós.

- CARPINTERO, ENRIQUE (1999). Registros de lo negativo. El cuerpo como lugar del inconsciente, el paciente límite y los nuevos dispositivos. Bs As. Editorial Topía.

- CHEMAMA, ROLAND; B. VANDERMERSCH Y OTROS, (2004). Diccionario de psicoanálisis. Buenos Aires. Amorrortu Editores.

- CIBEIRA, J. B. (2006). Aspectos Históricos del IREP. Visión de su primer director. En Boletín del Departamento de Docencia e Investigación del Instituto de Rehabilitación Psicofísica (IREP) Volumen 10 No 2 . Octubre 2006.

- FOUCAULT, MICHAEL (1992). La vida de los hombres infames. Buenos Aires, Editorial Altamira.

- FREUD, SIGMUND (1910). Sobre la perturbación psicógena de la visión. Buenos Aires. Amorrortu Editores.

- FREUD, S. (1915). Duelo y Melancolía. Buenos Aires. Amorrortu Editores.

- FREUD, S. (1923). El yo y el ello. Buenos
Aires. Amorrortu Editores.

- FREUD, S. (1924). El problema económico del masoquismo. Buenos Aires. Amorrortu Editores.

- FREUD, S. (1925). Algunas consecuencias psiquicas de la diferencia sexual anatómica. Buenos Aires. Amorrortu Editores.

- FREUD, S. (1937). Análisis terminable e interminable. Buenos Aires, Amorrortu Editores.

- LACAN, JACQUES (1966). Psicoanálisis y Medicina. En: Intervenciones y Textos 1. Buenos Aires, Manantial.

- LAPLANCHE Y PONTALIS (1971). Diccionario de psicoanálisis. Buenos Aires, Paidós.

- LEY N²6.378 (2008). Tratados internacionales. Convención Internacional sobre los Derechos de las Personas con Discapacidad y su protocolo facultativo. Beneficios jubilatorios. Argentina.

- ORGANizACIÓN DE LAS NACIONES UNIDAS (1982). Programa de Acción Mundial para las Personas con Discapacidad.

- ORGANIZACIÓN MUNDIAL DE LA SALUD (1997).Clasificación Internacional de las Deficiencias, Discapacidades y Minusvalias.

- PANTANO, LILIANA (1987). La discapacidad como problema social. Un enfoque sociológico, reflexiones y propuesta. Buenos Aires, Eudeba.

- PUig DE la BELlacAsa, RAMÓN (1990). Concepciones, Paradigmas y Evolución de las mentalidades sobre discapacidad. Ponencia presentada en el II Seminario sobre Discapacidad e información. Madrid. Mimeo.

- RABANT, CLAUDE (1993). Inventar lo real. Desestimación entre perversión y psicosis. Bs As. Editorial Nueva Visión

- RAIMBAULT, GINETTE (1985). El psicoanálisis y las fronteras de la medicina. Barcelona. Editorial Ariel.

- SACKS, OLIVER (2001). Un antropólogo en Marte. Siete relatos paradójicos. Madrid, Anagrama. 
- SIBILIA, PAULA (2005). El hombre postorgánico. Cuerpo, subjetividad y tecnologías digitales. Buenos Aires. Fondo de Cultura Económica .

- WASERMAN, MARIO (1995). Pensando en los trastornos del cuerpo. En Trastornos narcisistas no psicóticos. Estudios psicoanalíticos sobre problemáticas del cuerpo, el espacio y el aprendizaje en niños y adolescentes. Buenos Aires. Paidós. 